\title{
The Australian Complementary Medicine workforce: a profile of 1,306 practitioners from the PRACl study
}

Steel, Amie PhD ${ }^{1,2}$ (amie.steel@endeavour.edu.au)

Leach, Matthew PhD 2,3 (matthew.leach@unisa.edu.au)

Wardle, Jon PhD ${ }^{2}$ (jon.wardle@uts.edu.au)

Sibbritt, David PhD² (david.sibbritt@uts.edu.au)

Schloss, Janet PhD ${ }^{1}$ (janet.schloss@endeavour.edu.au)

Diezel, Helene Grad Dip Ed ${ }^{1}$ (janet.schloss@endeavour.edu.au)

Adams, Jon PhD ${ }^{1}$ (jon.adams@uts.edu.au)

${ }^{1}$ Office of Research, Endeavour College of Natural Health, Fortitude Valley, QLD Australia 4006

${ }^{2}$ Australian Research Centre in Complementary and Integrative Medicine, Faculty of Health, University of Technology Sydney, Ultimo, NSW Australia 2006

${ }^{3}$ University Department of Rural Health, University of South Australia, Adelaide, SA Australia 2001

Running head: Australian complementary medicine workforce 


\section{Abstract}

Objectives: This study aims to describe the Australian complementary medicine (CM) workforce including practice and professional characteristics.

Design: National, cross-sectional survey.

Settings/Location: Australia

Subjects: Any individual who self-identified as a practitioner qualified in any one of $14 \mathrm{CM}$ professions and worked in any State or Territory of Australia was eligible to participate in the survey.

Interventions: A 19 item online survey was developed following a review of existing CM workforce data and in alignment with other CM workforce survey projects in progress at the time. The survey items were presented under three main constructs: demographic characteristics, professional characteristics, and practice characteristics.

Statistical analysis: Descriptive statistical analysis, including frequencies and percentages, of multiple choice survey items was employed. Open response items were analysed to determine the mean, standard deviation (SD), minimum and maximum. The demographic data was evaluated for representativeness based on previously reported CM workforce figures.

Results: The survey was completed by $1306 \mathrm{CM}$ practitioners and was found to be nationally representative when compared with the most recent registrant data from the Chinese Medicine Board of Australia. Participants primarily practiced in the most populous Australian states and worked in at least one urban clinical location. Most participants held an Advanced Diploma qualification or lower, obtained their qualification ten more years ago and practiced in a clinical environment alongside at least one other practitioner from another health profession. Participants reported diverse clinical practice specialties and occupational roles. Per week, participants worked an average of 3.7 days and treated 23.6 clients.

Conclusions: The results from this survey of practitioners from most complementary professions in Australia provides new insights into the national complementary medicine workforce. Further exploration of the CM workforce is warranted to inform all who provide patient care and develop health policy for better patient and public health outcomes.

Keywords: Complementary therapies, health workforce, personnel 


\section{Introduction}

Complementary medicine (CM) - a cluster of treatments and therapies not traditionally taught or practised within conventional medicine ${ }^{1}-$ is used by a large proportion of the Australian population. ${ }^{2}$ This use is not limited to self-prescribed CM products and treatments, but also includes consultations with $\mathrm{CM}$ practitioners, ${ }^{2}$ with current data indicating that almost half of individuals with specific health diagnoses consults a CM practitioner for the management of their health condition. ${ }^{3-7}$ While consumer motivations for consulting $\mathrm{CM}$ practitioners have been well documented (i.e. alignment with holistic principles, ${ }^{8}$ patient-centred approach, ${ }^{9}$ patient education, patient empowerment) ${ }^{10}$ key information about the $\mathrm{CM}$ practitioner workforce, including the occupational roles undertaken by $\mathrm{CM}$ practitioners beyond the clinical encounter, is also under-examined.

\section{Diversity in occupational roles in the contemporary health workforce}

The health workforce is a large and diverse group of occupations primarily trained to work within clinical settings. The roles of these health providers can vary considerably, not only across occupational groups, but also within disciplines. ${ }^{11}$ This diversity may be driven by a number of forces, including practitioner-related issues (e.g. need for greater job satisfaction; opportunity for career advancement), patient-related issues (e.g. unmet community health care needs) and institutional context (e.g. neo-liberal management philosophies; greater emphasis on patient-led rather than practitioner-driven health services). ${ }^{11-14}$

Occupational role diversity can present a number of challenges for educational institutions, professional associations, regulatory bodies and policy makers. These challenges include the need to provide appropriate undergraduate and postgraduate training to adequately prepare practitioners for the various roles they may perform, ${ }^{15}$ and to ensure health professionals are acting appropriately within their intended scope of practice. This training is also paramount in ensuring health providers operate efficiently and effectively within the context of the broader health system. ${ }^{16,17}$

These challenges are potentially amplified in CM due to the lack of coherent professional leadership and variability often occurring in training within and across disciplines. ${ }^{18}$ Although some $\mathrm{CM}$ professions in Australia (i.e. traditional Chinese medicine, chiropractic and osteopathy) are under statutory registration, ${ }^{19}$ these are the exception, and the majority of $\mathrm{CM}$ professions are not formally registered by government. The unregistered CM professions exist largely beyond the gaze of those organizing health care provision and policy; reinforcing the view that CM operates as the 'black market of health care' ${ }^{20}$ In the case of unregistered CM professions, stakeholders have attempted to develop other mechanisms to compensate for the absence of statutory registration such as the National Code of Conduct for Health Care Workers ${ }^{21}$ and the Health Training Package developed by the federal and 
state governments, ${ }^{22}$ as well as practice standards and complaints mechanisms enacted through professional associations ${ }^{23,24}$ and independent registration bodies. ${ }^{25,26}$ Despite the mixed regulatory status of CM professions in Australia, the popularity of CM has not waned; in fact, the grass-roots movement toward an integrative model of health care has seen many health consumers choosing to consult a CM practitioner in conjunction with conventional healthcare providers. ${ }^{27,28}$

\section{The importance of workforce data to support policy and practice}

Government agencies responsible for providing accessible, affordable, efficient and safe health care to those in need rely upon accurate health workforce data to inform policy development. ${ }^{29}$ Equally, peak professional bodies charged with supporting the advancement, professionalisation and sustainability of a health profession benefit from detailed workforce data to assist them in their efforts. ${ }^{30}$ Some attempts have been made to characterise the Australian CM workforce by analysing existing data on the general $\mathrm{CM}$ workforce ${ }^{31}$ or by focusing on specific unregistered professions, such as Western herbalists, ${ }^{32-34}$ massage therapists, ${ }^{35}$ and naturopaths. ${ }^{32,34}$ This body of work has provided some insights into the demographic profile ${ }^{31,32}$ and attitudes of CM practitioners, ${ }^{35}$ as well as the practice characteristics of specific CM disciplines. ${ }^{32,33,35}$ Analyses of registrant data for the few CM professions that are registered in Australia, whilst representative of these professions, have largely been limited by focusing only on practitioner demographics and practice location ${ }^{36}$ with the chiropractic $^{37}$ and osteopathic ${ }^{38}$ professions the few exceptions. Only one of these studies captures a sample representative of the Australian population ${ }^{31}$ and while more accurate workforce data for registered professions can be found in government reports, ${ }^{30}$ there are still large areas of uncertainty regarding the details of the workforce for unregistered professions. Although investigations into the $\mathrm{CM}$ workforce have improved our understanding of this population, there are still many aspects that have yet to be examined (i.e. practice location, occupational roles, practice hours, patient load, etc.) ${ }^{39,40}$ the absence of such data limits the ability of policymakers to develop and implement responsive, appropriate and evidence-informed policies that impact the entirety of the health system..$^{29,41}$

The study reported here draws from national CM workforce survey data to address some of the knowledge gaps surrounding the Australian CM workforce; in doing so, the study aims to present a more comprehensive profile of the $\mathrm{CM}$ workforce in order to better inform future health workforce and health services planning.

\section{Materials and Methods}

\section{Design}

National, cross-sectional survey of Australian CM practitioners. 


\section{Aims and objectives}

This study aims to describe the Australian CM workforce including practice and professional characteristics.

\section{Sample}

Any individual who self-identified as a practitioner qualified in any one of $14 \mathrm{CM}$ professions (see Figure 1) and worked in any State or Territory of Australia was eligible to participate in the survey. Participants were required to have access to the internet as the survey was administered online. Chiropractors and osteopaths were excluded as other workforce surveys were known to be under development for both professions. ${ }^{42,43}$ The sample was recruited as part of the initiation of the Practitioner Research and Collaboration Initiative (PRACI); more information about the sample methodology has been described in detail previously. ${ }^{44}$ The minimum target sample size for statistical significance was determined to be 639 based on the most recent known number of CM practitioners $(n=16136)^{31}$ with a confidence level of $99 \%$ and a confidence interval of 5 .

\section{Survey}

The survey was following a review of existing CM workforce data and in alignment with other CM workforce survey projects in progress at the time. ${ }^{42}$ The draft survey was then piloted for face validity with six CM practitioners known to the research team from different professions and practice environments to ensure relevance to the diverse professional categories represented in the target sample. The survey comprised 19 items, which were presented under three main constructs: demographic characteristics (e.g. age, gender), professional characteristics (e.g. CM qualifications, years since first qualification, association membership, occupational roles) and practice characteristics (e.g. number of clients seen per week, practice location, number of clinics, other practitioners in primary clinical location, average rate charged per hour, clinical specialties [i.e. areas of special clinical interest or focus]). The survey items were primarily closed questions with multiple choice response options. Open questions were used for some questions targeting practice characteristics where the answer was expected to be a numeric value (e.g. average days worked per week, average clients seen per month, years in practice). The estimated completion time of the survey was 15 minutes.

\section{Data collection}

Eligible CM practitioners were identified using membership lists of professional $\mathrm{CM}$ associations (i.e. Australian Naturopathic Practitioners Association [ANPA], Naturopaths and Herbalists Association of Australia [NHAA], Massage and Myotherapy Australia [MMA], Australian Integrative Medicine Association [AIMA], Complementary Medicines Australia [CMA], Complementary Medicine Association [CMA], Australian Acupuncture and Chinese Medicine Association [AACMA], Australasian Association of Ayurevda [AAA], Australian Homoeopathic Association [AHA], Yoga Australia [YA], 
Reflexology Association of Australia [RAA], Association of Massage Therapists [AMT]) and customer lists of CM manufacturing companies (i.e. Health World Ltd, Bioconcepts Ltd, FIT-Bioceuticals Ltd, Naturopathic Products Online Database, Natural Therapies Pages). Two large professional associations did not choose to disseminate the survey invitation (Australian Natural Therapists Association [ANTA] and Australian Traditional Medicine Society]). However, through the client distribution lists of organisations such as the web-based service advertising site Natural Therapies Pages as well as practitioner-based supplement companies, we are confident that the invitation had a broad reach. Each organization was supplied with recruitment material and requested to communicate information about the study to $\mathrm{CM}$ practitioners on their database using whichever communication channel deemed appropriate to each organisation's management team. CM practitioners were also informed of the study using social media (e.g. Facebook, Twitter), and through practitioner meetings such as professional conferences. All recruitment material provided initial information about the project and a link to the survey. Upon accessing the survey link prospective participants were presented with the participant information sheet, which included an informed consent agreement and information about ethical clearance. The survey was administered online using the SurveyGizmo platform.

\section{Statistical analysis}

Data were exported from the SurveyGizmo platform into Stata $14^{\circledR}$ software for statistical analysis. Binary variables were created for items such as practice location and locality, professional qualifications, highest level of qualification held and practice special interests, to account for practitioners with multiple qualifications, practices and/or special interests. Descriptive analyses for all respondents, including frequencies and percentages, were calculated for variables of interest. Comparisons across professions were not possible, due to participants having qualifications across more than one profession. Chi-square analyses were used to evaluate the representativeness of the sample against demographic data for the Australian CM profession as published by Leach et al. ${ }^{31} \mathrm{An}$ additional analysis comparing respondents from the traditional Chinese medicine (TCM) professions (i.e. acupuncturists and Chinese herbalists) with national registration data ${ }^{30}$ was also undertaken, due to the limitations of Leach et al's CM workforce data. ${ }^{31}$

\section{Results}

\section{Demographic characteristics}

The survey was completed by 1,306 CM practitioners, the majority of whom were female ( $n=1,004$; 76.9\%) and aged between 40-59 years ( $n=775 ; 59.4 \%$ ). These figures were higher than those previously reported for the Australian CM workforce $(p<.001)$, but for TCM practitioners, were not significantly different to 2015 Chinese Medicine Board of Australia registrant data (see Table 1). 


\section{Practice characteristics}

The practice characteristics of respondents are presented in Table 2. Practitioners primarily practiced in the more populous Australian States of Victoria ( $n=454 ; 34.8 \%$ ), Queensland ( $n=317 ; 24.3 \%$ ) and New South Wales $(n=268 ; 20.5 \%)$, and notably less practiced in the smaller States and Territories. A small percentage of respondents also identified as having a 'virtual' clinical practice. While the majority of practitioners worked in a single clinical location $(n=848 ; 64.9 \%)$, more than one-third had two or more clinical practices. These clinics were predominantly located in an urban location ( $n=1067$; $81.7 \%)$, although at least one in four practitioners ( $n=325 ; 28.5 \%)$ had a clinic in a rural setting. Most respondents ( $n=1,291 ; 98.8 \%$ ) held at least one membership with a CM professional association.

There was wide variation in the number of years since participants had received their first CM-specific qualification, with most ( $n=868 ; 66.5 \%$ ) obtaining their qualification ten or more years ago (Table 2 ). There was also diversity in the composition of clinical teams, with $23.5 \%(n=304)$ working only in solo practice, $26.6 \%(n=345)$ working only in a shared clinical practice environment, and $49.9 \%(n=646)$ working across shared and solo practice settings. Chiropractors ( $n=109 ; 8.4 \%)$, counsellors $(n=102$; $7.8 \%$ ), physiotherapists $(n=92 ; 7.0 \%)$, and osteopaths ( $n=90,6.9 \%)$ were the professions that CM practitioners were most likely to share a clinical space with. CM practitioners were least likely to share a clinical space with midwives $(n=14 ; 1.1 \%)$, social workers $(n=23 ; 1.7 \%)$, specialist doctors $(n=26$; $2.0 \%)$ and community nurses $(n=31 ; 2.4 \%)$. Approximately $4.5 \%$ of respondents $(n=59)$ were colocated with a general practitioner.

CM practitioners reported twenty different clinical practice specialties (Table 2). The most frequently reported specialties were general health and wellbeing ( $n=949 ; 72.7 \%$ ), musculoskeletal conditions ( $n=921 ; 70.5 \%)$ and pain management $(n=778 ; 59.6 \%)$. Practice specialties reported the least were paediatrics $(n=393 ; 30.1)$, renal health $(n=342 ; 26.2 \%)$ and gerontology $(n=252 ; 19.3 \%)$.

The outcomes of the analysis regarding clinical consultation economics is reported in Table 3. The respondents worked an average of 3.7 days per week as a CM practitioner, with naturopaths working the least number of days per week (mean: 3.2 days) and myotherapists working the greatest number of days per week (mean: 4.3 days). These differences did not directly translate to the number of client hours worked per week, with the lowest number of hours worked reported by reflexologists (mean: 13.9 hours/week), and the highest number reported by Chinese herbalists (mean: 25.5 hours); the average for all respondents was 17.8 hours per week.

The mean number of clients consulted per week was also greatest for Chinese herbalists (28.4 clients), but the lowest number was reported by Ayurvedic practitioners ( 9.7 clients). The average number of new clients seen per month for all CM professions was 23.6 clients. Chinese herbal medicine 
practitioners and acupuncturists reported a similar, and equally high number of new clients per month (mean: 43.2 clients), compared to reflexologists who reported seeing the least (mean: 14.7 clients). The average rate charged per hour as a CM practitioner was $\$ 83.58$, although this varied across professional categories, with homoeopaths reporting the highest hourly rate (mean: \$106.57) and reflexologists reporting the lowest hourly rate (mean: \$71.78).

\section{Professional characteristics}

The majority of respondents identified as being in clinical practice as a CM practitioner ( $n=1184$; 90.7\%) and spending on average $80 \%$ (SD 28.4) of their work time in this role (Table 4). Participants had worked a mean 5.9 (SD 7.8) years full-time and 5.7 (SD 6.6) years part-time as a CM practitioner. In addition to CM clinical practice, $19.7 \%(n=251)$ also worked in a non-CM clinical role for approximately 52.1\% (SD 32.1) of their work time. A similar number of respondents reported other occupational roles such as lecturer $(18.5 \% ; n=156)$, group educator $(9.4 \%, n=123)$ and retail employee $(9.3 \%, n=71)$. For those with a non-clinical occupational role, the average percentage of time committed to this role ranged between $19.4 \%$ (group educator, SD 21.6 ) and $42.0 \%$ (retail employee, SD 26.8).

In terms of practitioner qualifications, the majority of participants across most professions held an Advanced Diploma qualification or lower, with the only exceptions being acupuncture and Chinese herbal medicine (Table 5). Most acupuncturists held a Bachelor degree qualification ( $n=112 ; 64.7 \%$ ), with almost one in four $(n=32 ; 18.5 \%)$ possessing a postgraduate qualification. More than half $(n=45$; $52.9 \%$ ) of Chinese herbalists had a Bachelor degree qualification, with almost one-third ( $n=24 ; 28.2 \%$ ) holding a postgraduate qualification. Although two-thirds $(n=193 ; 66.8 \%)$ of naturopaths had an Advanced Diploma qualification or less, there was still a sizeable proportion that held a Bachelor degree qualification ( $n=123 ; 42.6 \%)$; however, very few $(n=7 ; 2.4 \%)$ had a postgraduate qualification. By contrast, while a similar proportion of clinical nutritionists also held an Advanced Diploma qualification or less $(n=113 ; 68.1 \%)$, a relatively greater proportion of this group had a postgraduate qualification ( $n=18 ; 10.8 \%)$. Of all professions, aromatherapists $(98.2 \%)$ and reflexologists $(97.5 \%)$ had the largest proportion of respondents with an Advanced Diploma qualification or lower.

\section{Discussion}

This study highlights the diversity of the CM workforce in Australia and offers an update to existing workforce data as well as offering new insights into the non-clinical roles undertaken by CM practitioners. The study makes a number of important observations; firstly, although most Australian CM practitioners work as clinicians, a sizeable proportion of this workforce are also engaging in other non-clinical roles. While previous research has identified diversity in the types of treatments and 
therapies employed in the $\mathrm{CM}$ clinical setting, ${ }^{32}$ this research underlines the variety of settings and roles outside of clinic that comprise a CM practitioner's occupational life. Whether this mixture of roles is influenced by transitions in the CM practitioner's career ${ }^{11}$ (i.e. transitioning from study to practice; establishing a new practice; responding to patient-led demand for health services or unmet community health need $)^{11-13}$ is not yet clear.

Some CM practitioners report working as retail employees, most likely in pharmacy or health food store settings. ${ }^{45}$ This occupational role may be driven by consumer demand as previous research indicates that $58 \%$ of pharmacy customers endorse the employment of a $\mathrm{CM}$ practitioner in pharmacies that stock complementary medicines ${ }^{46}$ Public safety concerns also have been raised by researchers, as untrained health food store assistants have been found to provide unsafe health care advice to customers. ${ }^{47}$ Alternatively, or perhaps in addition to the possible market demand for $\mathrm{CM}$ qualified retail staff, practitioners may be choosing to integrate retail duties into their occupation to address insufficiencies in their income from clinical practice. ${ }^{32}$ While there is emerging evidence regarding the nature of health advice provided to customers of health food stores ${ }^{48}$ and pharmacies, ${ }^{49}$ there has been little exploration of the role and contribution of qualified CM practitioners within those retail environments, pointing to a need for additional research in this area.

The number of $\mathrm{CM}$ practitioners working as group educators lends weight to the argument that some CM professions practice in accordance with, or are philosophically aligned with, the principles of public health and health promotion. ${ }^{50,51}$ While existing research suggests that $\mathrm{CM}$ practitioners emphasise patient education within the clinical consultation ${ }^{9,10}$ and may focus on wellness and disease prevention, ${ }^{52}$ this is the first known study to reveal that these professions also provide group education to the community. However, the content of these CM-practitioner led group education sessions is not yet known, and warrants closer examination before the role of CM practitioners as providers of community health promotion and public health services can be assumed. Overall, this study offers new insights into the roles that CM practitioners play within the health system, but urges caution in reaching any firm conclusions until a more comprehensive exploration of these roles has been undertaken.

The findings of this study also suggest that Australian CM practitioners have diverse practice specialties. While the most common practice specialty reported by respondents in this study was 'general health and wellbeing', other more specific disease-oriented interest areas were also common. The relationship between $\mathrm{CM}$ and wellness is well-established both at a theoretical and philosophical level ${ }^{53}$ as well as in the real-world; ${ }^{54}$ it is also a key reason why individuals report using $\mathrm{CM}$. However, although CM is known to be used for specific conditions such as cancer, ${ }^{7,55}$ depression $^{6}$ 
and arthritis, ${ }^{4}$ the specific role and contribution of $\mathrm{CM}$ practitioners in the management of these and other health conditions are largely unknown. ${ }^{39,40}$ In fact, the degree to which CM practitioners valueadd clinical care has received sporadic interest from researchers at best. ${ }^{52,56}$ This study therefore provides a useful direction for future researchers seeking to explore this topic more carefully.

Another observation of this study is the considerable variation in the level of training of Australian CM practitioners. This variation most likely reflects the availability of university training for $\mathrm{CM}$ in Australia. ${ }^{57}$ Notwithstanding, it is unlikely that access to professional education is the only contributing factor, as almost half of naturopaths reported having an Advanced Diploma qualification or less, despite a Bachelor degree qualification for naturopathy being available in Australia for over twenty years. ${ }^{18}$ Statutory regulation also may have an influential effect on the level of practitioner training within specific disciplines, with the two statutory regulated professions in this study (i.e. Acupuncture and Chinese herbal medicine) both reporting the highest proportion of Bachelor degree or postgraduate qualified practitioners. ${ }^{36,58}$

Many of the metrics associated with economic viability of $\mathrm{CM}$ clinical practice (i.e. days worked per week, number of clients seen per week, number of new clients per month) were above average for most CM professions in this study that are characterised by 'practitioner-administered' treatments such as acupuncture and massage therapy. ${ }^{16}$ Many of the CM professions that deliver 'practitioneradministered treatments' may also be benefiting from high rates of referral from other health professionals. ${ }^{59}$ By contrast, the professions that primarily provided patient-administered treatments, ${ }^{10}$ such as naturopathy and homeopathy, charged above average hourly rates. Given that the factors driving the economics of health service delivery are reasonably complex, the reasons for these differences are not entirely clear. It may be that those CM professions in this study with a higher average hourly rate are driven by a need to maintain practice viability in response to decreasing patient numbers. ${ }^{60}$ It is also possible that practitioners in professions characterized by prescription of patient-administered treatments may be responding to increased market demand due to the relative size of their workforce. ${ }^{61}$

Contrary to reports from previous studies that Australian CM practitioners work alone and are isolated from health care teams ${ }^{32}$ the findings of this study indicate that three out of every four Australian CM practitioners provide consultations in a shared clinical environment of some kind. This difference may be due to the evolving professionalization and mainstreaming of $\mathrm{CM}$ in the years since this previous research was conducted. However, this pattern of shared clinical location was less likely to be inclusive of medical doctors such as GPs or specialist doctors; suggesting that CM practitioners are still practicing at structural and geographical distances from the core of mainstream health care delivery 
in Australia. Furthermore, it is not clear whether this finding reflects interprofessional collaboration or just co-location. There are diverse models of care reported in the literature within the broad scope of integrative or complementary medicine, ${ }^{27}$ with several studies exploring the interprofessional communication between $\mathrm{CM}$ practitioners and other health professions reporting varying success. ${ }^{32,62,63}$ and as such suggest that, along with the level of isolation of CM within the broader health system, the practice environments for $\mathrm{CM}$ practitioners may be adapting to the changing role of $\mathrm{CM}$ in contemporary healthcare. It is interesting to note that the health professionals most commonly sharing a clinical space with CM practitioners in this study provided either manual therapies or psychological support. As a possible explanation of this trend, the importance of mind-bodymedicine is acknowledged within $\mathrm{CM}$ as a core component of holistic philosophy ${ }^{8}$ and may compel $\mathrm{CM}$ practitioners to provide patient referrals to health professionals that are able to provide more advanced and specialized support for their patients' emotional and physical health complaints. Closer researcher attention to the structural, spatial and interpersonal interface between $\mathrm{CM}$ and other health professionals is needed before any of these possible interpretations can be verified.

\section{Limitations}

While contributing to our understanding of the Australian $\mathrm{CM}$ workforce, there are limitations to this study that require consideration. Firstly, the representativeness of the data is not clear. Based on comparisons with Australian Census data ${ }^{31}$, our sample appeared to be older and more femaledominant than the national average; however, it is possible that the exclusion of chiropractors and osteopaths in our sample (both male-dominant professions) ${ }^{31}$, and the age of the census data (i.e. more than ten years old) may have contributed to the differences between data sets. According to more recent data (i.e. CMBA registrant data), ${ }^{58}$ our sample of TCM practitioners does appear to be nationally representative. As such, while our analysis does suggest our sample may be nationally representative, no firm conclusions on this issue can be drawn. Our study is also limited by factors inherent in the study design, such as the risk of response bias, as all answers were self-reported.

\section{Conclusions}

$\mathrm{CM}$ use, including consultations with $\mathrm{CM}$ practitioners, is widespread and the $\mathrm{CM}$ workforce provides substantial levels of clinical care in many important areas of health. The characteristics of CM practice is diverse and reflects clear differences in practice dynamics and economics between the various CM professions. CM practitioners are also fulfilling a more diverse range of occupational roles than has been previously considered. The implications of this apparent diversity in CM practice within and across CM professions warrants further exploration in order to help inform all stakeholders involved in providing patient care and developing health policy for better patient and public health outcomes. 


\section{Acknowledgments}

The PRACl project is funded by Endeavour College of Natural Health and supported by Key Support Partners including Massage and Myotherapy Australia, Complementary Medicines Australia, Australian Homeopathic Association, Health World Ltd, and FIT-Bioceuticals Ltd.

Author disclosure statement

The authors have not conflicts of interest to disclose. 
1. Adams J. Introduction. In: Adams J, ed. Researching Complementary and Alternative Medicine. Oxon: Routeledge; 2007: xiii-xx.

2. Reid R, Steel A, Wardle J, Trubody A, Adams J. Complementary medicine use by the Australian population: a critical mixed studies systematic review of utilisation, perceptions and factors associated with use. BMC Complementary and Alternative Medicine 2016; 16(1): 176. 3. Murthy V, Sibbritt D, Adams J, Broom A, Kirby E, Refshauge KM. Consultations with complementary and alternative medicine practitioners amongst wider care options for back pain: a study of a nationally representative sample of 1,310 Australian women aged 60-65 years. Clinical rheumatology 2014; 33(2): 253-62.

4. Murthy V, Sibbritt DW, Adams J. An integrative review of complementary and alternative medicine use for back pain: a focus on prevalence, reasons for use, influential factors, self-perceived effectiveness, and communication. The Spine Journal 2015; 15(8): 1870-83.

5. Steel A, Adams J, Sibbritt D, Broom A, Gallois C, Frawley J. Utilisation of complementary and alternative medicine (CAM) practitioners within maternity care provision: results from a nationally representative cohort study of 1,835 pregnant women. BMC Pregnancy Childbirth, 2012. (accessed. 6. Solomon D, Adams J. The use of complementary and alternative medicine in adults with depressive disorders. A critical integrative review. Journal of affective disorders 2015; 179: 101-13. 7. Truant T, Porcino A, Ross B, Wong M, Hilario C. Complementary and alternative medicine (CAM) use in advanced cancer: a systematic review. The journal of supportive oncology 2013; 11(3): 105-13.

8. Foley $\mathrm{H}$, Steel A. The Nexus Between Patient-Centered Care and Complementary Medicine: Allies in the Era of Chronic Disease? The Journal of Alternative and Complementary Medicine 2017; 23(3): 158-63.

9. Foley $\mathrm{H}$, Steel A. Patient perceptions of clinical care in complementary medicine: A systematic review of the consultation experience. Patient Education and Counseling 2016.

10. Foley $\mathrm{H}$, Steel A. Patient perceptions of patient-centred care, empathy and empowerment in complementary medicine clinical practice: A cross-sectional study. Advances in Integrative Medicine 2017.

11. Nancarrow SA, Borthwick AM. Dynamic professional boundaries in the healthcare workforce. Sociology of health \& illness 2005; 27(7): 897-919.

12. Collins K, Jones M, Mcdonnell A, Read S, Jones R, Cameron A. Do new roles contribute to job satisfaction and retention of staff in nursing and professions allied to medicine? Journal of Nursing Management 2000; 8(1): 3-12.

13. Stanhope M, Lancaster J. Public health nursing: Population-centered health care in the community: Elsevier Health Sciences; 2015.

14. Leach MJ. Using role substitution to address the health workforce shortage and to facilitate integration? Journal of Complementary and Integrative Medicine 2012; 9(1).

15. Overton A, Clark M, Thomas Y. A review of non-traditional occupational therapy practice placement education: a focus on role-emerging and project placements. British Journal of Occupational Therapy 2009; 72(7): 294-301.

16. Kleinman CS. Leadership roles, competencies, and education: how prepared are our nurse managers? Journal of Nursing Administration 2003; 33(9): 451-5.

17. Pulcini J, Jelic M, Gul R, Loke AY. An international survey on advanced practice nursing education, practice, and regulation. Journal of Nursing Scholarship 2010; 42(1): 31-9.

18. Wardle J, Steel A, Adams J. A review of tensions and risks in naturopathic education and training in Australia: a need for regulation. J Altern Complement Med 2012; 18(4): 363-70.

19. Australian Health Practitioner Regulation Agency. Welcome to AHPRA. 2012. http://www.ahpra.gov.au/ (accessed 10/07/2012. 
20. Wardle J. Complementary and integrative medicine: The black market of health care? : Elsevier; 2016.

21. COAG Health Council. National Code of Conduct for Health Care Workers. 2015 (accessed 6 Sep 2017).

22. Training.gov.au. HLT - Health (Release 3.1). In: Governments SaF, editor.:

https://training.gov.au/Training/Details/HLT; 2016.

23. Naturopaths and Herbalists Association of Australia. Education. 2017 (accessed 6 Sep 2017).

24. Massage and Myotherapy Australia. Code of Ethics. 2017 (accessed 6 Sep 2017).

25. Australian Register of Homeopaths. Policies \& Guidelines. 2017 (accessed 6 Sep 2017).

26. Australian Register of Naturopaths and Herbalists. ARONAH Documents. 2017 (accessed 6 Sep 2017).

27. Lim EJ, Vardy JL, Oh BS, Dhillon HM. A Scoping Review on Models of Integrative Medicine:

What Is Known from the Existing Literature? The Journal of Alternative and Complementary Medicine 2017; 23(1): 8-17.

28. Leach MJ. Profiling the Australian Consumer of Complementary and Alternative Medicine: A Secondary Analysis of National Health Survey Data. Alternative therapies in health and medicine 2016; 22(4): 64.

29. Beaglehole R, Dal Poz MR. Public health workforce: challenges and policy issues. Human resources for health 2003; 1(1): 4.

30. Balasubramanian M, Brennan D, Spencer A, Watkins K, Short S. The importance of workforce surveillance, research evidence and political advocacy in the context of international migration of dentists. British dental journal 2015; 218(6): 329-31.

31. Leach MJ. Profile of the complementary and alternative medicine workforce across Australia, New Zealand, Canada, United States and United Kingdom. Complementary therapies in medicine 2013; 21(4): 364-78.

32. Bensoussan A, Myers S, Wu S, O'Connor K. Naturopathic and Western Herbal Medicine practice in Australia - a workforce survey. Complement Ther Med 2004; 12: 17-27.

33. Casey MG, Adams J, Sibbritt D. An examination of the prescription and dispensing of medicines by Western herbal therapists: A national survey in Australia. Complement Ther Med 2007; 15(1): 13-20.

34. Leach MJ, McIntyre E, Frawley J. Characteristics of the Australian complementary and alternative medicine (CAM) workforce. Australian Journal of Herbal Medicine 2014; 26(2): 58.

35. Wardle JL, Barnett R, Adams J. Practice and research in Australian massage therapy: a national workforce survey. International journal of therapeutic massage \& bodywork 2015; 8(2): 2.

36. Chinese Medicine Board of Australia. Registrant Data.

http://www.chinesemedicineboard.gov.au/About/Statistics.aspx: Chinese Medicine Board of Australia, 2015.

37. Adams J, Lauche R, Peng W, et al. A workforce survey of Australian chiropractic: the profile and practice features of a nationally representative sample of 2,005 chiropractors. BMC complementary and alternative medicine $2017 ; 17(1)$ : 14.

38. Orrock P. Profile of members of the Australian Osteopathic Association: part 1-the practitioners. International Journal of Osteopathic Medicine 2009; 12(1): 14-24.

39. Steel A, Adams J. The role of naturopathy in pregnancy, labour and postnatal care: broadening the evidence-base. Complement Ther Clin Pract 2011; 17: 2011.

40. Steel A, Blaich R, Sundberg T, Adams J. The role of osteopathy in clinical care: Broadening the evidence-base. International Journal of Osteopathic Medicine 2017; 24: 32-6.

41. Steel A, Adams J, Frawley J, et al. Does Australia's Health Policy Environment Create Unintended Outcomes for Birthing Women? Birth 2016; 43(4): 273-6.

42. Adams J, Steel A, Moore C, Amorin-Woods L, Sibbritt D. Establishing the ACORN national practitioner database: Strategies to recruit practitioners to a national Practice-Based Research Network. Journal of manipulative and physiological therapeutics 2016; 39(8): 594-602. 
43. Australian Research Centre in Complementary and Integrative Medicine. Introduction to ORION. 2017. http://www.orion-arccim.com/ (accessed 5 Jul 2017).

44. Steel A, Adams J, Sibbritt D. Developing a multi-modality complementary medicine practicebased research network: The PRACI project. Advances in Integrative Medicine 2014; 1(3): 113-8.

45. Braun LA, Spitzer O, Tiralongo E, et al. The prevalence and experience of Australian naturopaths and Western herbalists working within community pharmacies. BMC complementary and alternative medicine 2011; 11(1): 41.

46. Braun LA, Tiralongo E, Wilkinson JM, et al. Perceptions, use and attitudes of pharmacy customers on complementary medicines and pharmacy practice. BMC Complementary and Alternative Medicine 2010; 10(1): 38.

47. Glisson JK, Rogers HE, Abourashed EA, Ogletree R, Hufford CD, Khan I. Clinic at the health food store? Employee recommendations and product analysis. Pharmacotherapy: The Journal of Human Pharmacology and Drug Therapy 2003; 23(1): 64-72.

48. Calder J, Issenman R, Cawdron R. Health information provided by retail health food outlets. Canadian Journal of Gastroenterology and Hepatology 2000; 14(9): 767-71.

49. Cramer H, Shaw A, Wye L, Weiss M. Over - the - counter advice seeking about complementary and alternative medicines (CAM) in community pharmacies and health shops: an ethnographic study. Health \& social care in the community 2010; 18(1): 41-50.

50. Giordano J, Garcia MK, Boatwright D, Klein K. Complementary and alternative medicine in mainstream public health: a role for research in fostering integration. The Journal of Alternative \& Complementary Medicine 2003; 9(3): 441-5.

51. Wardle J, Oberg EB. The Intersecting Paradigms of Naturopathic Medicine and Public Health: Opportunities for Naturopathic Medicine. J Altern Complement Med 2012; 17(11): 1079-84.

52. Oberg E, Bradley R, Cooley K, Fritz H, Goldenberg J. Estimated Effects of Whole-system Naturopathic Medicine in Select Chronic Disease Conditions: A Systematic Review. Altern Integ Med 2015; 4(192): 2.

53. Schuster TL, Dobson M, Jauregui M, Blanks RH. Wellness lifestyles I: A theoretical framework linking wellness, health lifestyles, and complementary and alternative medicine. The Journal of Alternative \& Complementary Medicine 2004; 10(2): 349-56.

54. Hawk C, Ndetan $\mathrm{H}$, Evans MW. Potential role of complementary and alternative health care providers in chronic disease prevention and health promotion: an analysis of National Health Interview Survey data. Preventive medicine 2012; 54(1): 18-22.

55. Bao $Y$, Kong $X$, Yang $L$, et al. Complementary and alternative medicine for cancer pain: an overview of systematic reviews. Evidence-Based Complementary and Alternative Medicine 2014; 2014.

56. Steel A, Sundberg T, Reid R, et al. Osteopathic manipulative treatment: A systematic review and critical appraisal of comparative effectiveness and health economics research. Manual Therapy 2016.

57. Steel A, McEwen B. The need for higher degrees by research for complementary medicine practitioners. Australian Journal of Herbal Medicine 2014; 26(4): 136.

58. Chinese Medicine Board of Australia. Chinese Medicine Board of Australia. 2012. http://www.chinesemedicineboard.gov.au/ (accessed 18/07/2012.

59. Wardle JL, Adams J, Sibbritt DW. Acupuncture in Australian general practice: trends in reimbursed acupuncture services from 1995 to 2011. Acupuncture in Medicine 2013; 31(1): 45-50.

60. Sibbritt $D$. The decline of herbal medicine/naturopathy consultations: How research can help further the profession. Australian Journal of Herbal Medicine 2014; 26(1): 8.

61. Wardle J, Adams J, Magalhães RJS, Sibbritt D. Distribution of complementary and alternative medicine (CAM) providers in rural New South Wales, Australia: A step towards explaining high CAM use in rural health? Aust J Rural Health 2011; 19(4): 197-204. 
62. Diezel H, Steel A, Wardle J, Johnstone K. Patterns and influences of interprofessional communication between midwives and CAM practitioners: a preliminary examination of the perceptions of midwives. Aust J Herbal Med 2013; 25(1): 4-10.

63. Steel A, Diezel H, Wardle J, Johnstone K. Patterns of inter-professional communication between complementary and conventional practitioners providing maternity care services: a preliminary examination of the perceptions of CAM practitioner. Australian Journal of Herbal Medicine 2013; 25(2).

Corresponding author:

Dr Amie Steel

Level 2, 269 Wickham St

Fortitude Valley

QLD Australia 4006

Amie.steel@endeavour.edu.au 